\title{
Inference on the Parameters and Optimal Design of Simple Step-Stress Accelerated Life Testing for Weighted Exponential Distribution
}

\author{
Hamdy M. Salem \\ Department of Statistics - Faculty of Commerce - Al-Azher University - Egypt \\ E-mail (d.hamdysalm@yahoo.com ).
}




\begin{abstract}
:
This paper considers life-testing experiments and its influence of stress factors such as, temperature or electricity loads on the lives of experiment items. Step-Stress test is a special class of accelerated life tests, which allows the researcher to increase stress levels more than normal during the experiment to see the surviving items. The lifetime distribution of the test items is assumed to follow weighted exponential distribution that considered. Different methods for estimating the parameters are discussed. These methods are mainly maximum likelihood and confidence interval estimations which generates narrow intervals to the unknown parameters of the distribution with high probability based on asymptotic normality. Numerical study to illustrate the optimal time procedure using MathCAD (2001) is discussed.
\end{abstract}

Keywords: Accelerate Life Testing, Step-Stress, Maximum Likelihood Estimator; Information Matrix, Confidence Interval Estimators, Weighted Exponential Distributions.

\title{
1. Introduction
}

The Accelerated Life Tests play an important role in reliability analysis. The stress levels are increasing discretely at pre-fixed time points on test units during an experiment. This is called step-stress test. Under normal operating conditions, it allows the experimenter to obtain information on the parameters of the lifetime distributions readily. In addition, when a test unit fails, there are many causes for the failure such as mechanical, temperature and electrical.

Many authors have measured different choices for accelerate life testing. Drop, R. V. et al (1996) developed a Bayes model for step-stress accelerated life testing. They assumed the failure times at each stress level are having exponential distribution but the specification of strict compliance to a time transformation function is not required. Dharmadhikari, A. D. \& Monsur M. R. (2003) assumed that the scale parameter of Weibull and lognormal models which depend upon the present level as well as the age at the entry in the present stress. They proposed a parametric model to the life distribution for step-stress testing and estimated the parameters involved in it. Chen, Z. et al (2006) considered the step-stress 
accelerated life tests for exponential population. They applied the tampered failure rate model to be evidence for the existence, uniqueness, strong consistency and the asymptotic normality of the MLE of mean. Al-Ghamdi A. S. \& Hassan, A. S. (2009) considered the problem using the Lomax distribution under a cumulative exposure model. They obtained the optimum test plan by minimizing with respect to the change time scale parameter and the asymptotic variance of the maximum likelihood estimator of a given 50th percentile.

Recently, Donghoon, H. \& Balakrishnan, N. (2010) considered the simple step-stress model under time constraint when the lifetime distributions of the different risk factors are independently and exponentially distributed. they derived the maximum likelihood estimators of the unknown mean parameters under the assumption of a cumulative exposure model. . Abd-Elfattah, A. M. and Al-Harbey, A. H. (2010) studied the estimation problem when the lifetime distribution of the test items is following Burr type III distribution. They derived the Maximum Likelihood Estimates for the distribution parameters and acceleration factor in type II censored samples. Lee, H. et al (2013) studied the problem for exponential products based on type II right censored data from the step-stress accelerated life test. He preformed tests of hypotheses about model parameters based on the likelihood method.

Azzalini (1985) suggested a method of obtaining weighted distributions from independently identically distributed random variables. He used the density function of one random variable and the distribution function of the other random variable as follows:

$$
F_{X}(x)=\frac{1}{P\left(\alpha X_{1}>X_{2}\right)} f_{Y}(x) F_{Y}(\alpha x)
$$

Gupta \& Kundu, (2009) presented a new class of weighted exponential distributions based on an idea of Azzalini (1985). Makhdoom (2012) studied the estimation of Stress-Strength reliability when $\mathrm{X}$ and $\mathrm{Y}$ are two weighted exponential distributions with different parameters. He obtained the MLE of $\mathrm{R}$ based on one simple iteration procedure, and he carried out Bayesian estimators of parameters with real data.

The cumulative distribution function and the probability density function of a random variable which has the weighted exponential distribution respectively are: 


$$
\begin{aligned}
& F(x ; \alpha, \lambda)=\frac{\alpha+1}{\alpha}\left[1-e^{-\lambda x}-\frac{1}{\alpha+1}\left(1-e^{-(\alpha+1) \lambda x}\right)\right] \quad ; \alpha, \lambda, x>0, \\
& f(x ; \alpha, \lambda)=\frac{\alpha+1}{\alpha} \cdot \lambda e^{-\lambda x}\left(1-e^{-\alpha \lambda x}\right) \quad ; \alpha, \lambda, x>0 .
\end{aligned}
$$

hence $\alpha$ and $\lambda$ are the shape and scale parameters respectively (see Gupta \& Kundu 2009). This paper consists of four sections corresponding to sections 2, 3, 4 and 5, respectively. section 2 discusses the model description and assumptions. section 3, provides the likelihood function and the asymptotic Fisher information matrix which is contain on second and partial derivatives of the logarithm of likelihood function. Confidence intervals about model parameters is examined based on asymptotic normality in chapter 4 . Finally, the last section contains a numerical investigation carried out to study the properties of the new estimators.

\section{Model Description and Assumptions}

The cumulative distribution function of weighted exponential distribution which is mentioned in(1.1) contains failure time of test item in case constant stress. and the assumptions made are as below:

1- There is a relation that represents the relation between stress level $Z_{i}$ and scale parameter $\lambda_{i}$ as follows:

$\log \left(\lambda_{i}\right)=\theta_{0}+\theta_{1} Z_{i} \quad i=1,2$ where $\theta_{0}, \theta_{1}$ are unknown parameters depend on the way and outcomes of the test and.

2- The entire $n_{i}, \quad i=1,2$ items are put at the normal stress $Z_{1}$, at the beginning, continuing until time $\tau$. Next, the stress is varied to a high level $Z_{2}$, and the test ongoing until all items failed.

3- At the time $x_{i j}, j=1,2, \ldots, n_{i}$ under a stress level $Z_{i}, i=1,2$, all of $n_{i}$ failure are observed.

4- In Step-Stress test which is a special class of accelerated life tests, the cumulative exposure model is:

$$
F(x)=\left\{\begin{array}{cl}
F_{1}(x) & 0<x<\tau \\
F_{2}\left(x-\tau+\tau^{\prime}\right) & \tau<x<\infty
\end{array}\right.
$$


where $\tau^{\prime}=\tau\left(\frac{\lambda_{2}}{\lambda_{1}}\right)$ which is asolution of $F_{1}(\tau)=F_{2}\left(\tau^{\prime}\right)$ for $\tau^{\prime}$. So the cumulative exposure model for weighted exponential distributions is obtained as follow:

$$
F(x)= \begin{cases}\frac{\alpha+1}{\alpha}\left[1-e^{-\lambda_{1} x}-\frac{1}{\alpha+1}\left(1-e^{-(\alpha+1) \lambda_{1} x}\right)\right] & ; 0 \leq x<\tau \\ \frac{\alpha+1}{\alpha}\left[1-e^{-\lambda_{2}\left(x-\tau\left(1-\frac{\lambda_{2}}{\lambda_{1}}\right)\right)}-\frac{1}{\alpha+1}\left(1-e^{-(\alpha+1) \lambda_{2}\left(x-\tau\left(1-\frac{\lambda_{2}}{\lambda_{1}}\right)\right)}\right)\right] & ; \tau \leq x<\infty\end{cases}
$$

and the probability density function of the failure time is:

$$
f(x)= \begin{cases}\frac{\alpha+1}{\alpha} \cdot \lambda_{1} e^{-\lambda_{1} x}\left(1-e^{-\alpha \lambda_{1} x}\right) & ; 0 \leq x<\tau \\ \frac{\alpha+1}{\alpha} \cdot \lambda_{2} e^{-\lambda_{2}\left(x-\tau\left(1-\frac{\lambda_{2}}{\lambda_{1}}\right)\right)\left(1-e^{-\alpha \lambda_{2}\left(x-\tau\left(1-\frac{\lambda_{2}}{\lambda_{1}}\right)\right)}\right)} & ; \tau \leq x<\infty\end{cases}
$$

In next chapter, the likelihood function and asymptotic Fisher information matrix are provided.

\section{Likelihood Function and Asymptotic Fisher Information Matrix}

Let the failure times $x_{i j}, j=1,2, \ldots, n_{i}, i=1,2$ and $n_{1}, n_{2}$ are the number of items which faile at normal stress $Z_{1}$ and high stress $Z_{2}$ respectively. Then, their likelihood function and log likelihood function respectively are:

$$
\begin{aligned}
L\left(x ; \alpha, \lambda_{1}, \lambda_{2}\right)= & \left(\frac{\alpha+1}{\alpha}\right)^{n_{1}}\left(\frac{\alpha+1}{\alpha}\right)^{n_{2}} \lambda_{1}^{n_{1}} \lambda_{2}^{n_{2}} \prod_{j=1}^{n_{1}} e^{-\lambda_{1} x_{1 j}} \prod_{j=1}^{n_{1}}\left(1-e^{-\alpha \lambda_{1} x}\right) \\
& \times \prod_{j=1}^{n_{1}} e^{-\lambda_{2}\left(x_{2 j}-\tau\left(1-\frac{\lambda_{2}}{\lambda_{1}}\right)\right)} \prod_{j=1}^{n_{2}}\left(1-e^{\left.-\alpha \lambda_{2}\left(x_{2 j}-\tau\left(1-\frac{\lambda_{2}}{\lambda_{1}}\right)\right)\right),}\right. \\
\ell\left(x ; \alpha, \lambda_{1}, \lambda_{2}\right)= & \left(n_{1}+n_{2}\right) \log (\alpha+1)-\left(n_{1}+n_{2}\right) \log (\alpha) \\
& -\lambda_{1} x_{1 j}-\lambda_{2}\left(x_{2 j}-\tau\left(1-\frac{\lambda_{2}}{\lambda_{1}}\right)\right)+\sum_{j=1}^{n_{1}} \log \left(1-e^{-\alpha \lambda_{1} x_{1 j}}\right) \\
& +\sum_{j=1}^{n_{2}} \log \left(1-e^{-\alpha \lambda_{2}\left(x_{2 j}-\tau\left(1-\frac{\lambda_{2}}{\lambda_{1}}\right)\right)}\right) .
\end{aligned}
$$


The first derivatives of $\ell\left(x ; \alpha, \lambda_{1}, \lambda_{2}\right)$ with respect to $\alpha, \lambda_{1}$ and $\lambda_{2}$ are, respectively:

$$
\begin{aligned}
& \frac{\partial \ell\left(x ; \alpha, \lambda_{1}, \lambda_{2}\right)}{\partial \alpha}=-\frac{\left(n_{1}+n_{2}\right)}{\alpha(\alpha+1)}+\lambda_{1} \sum_{j=1}^{n_{1}} x_{1 j} t^{*} \\
& +\lambda_{2} \sum_{j=1}^{n_{1}}\left(x_{2 j}-\tau\left(1-\frac{\lambda_{2}}{\lambda_{1}}\right)\right) \cdot t * *=0, \\
& \frac{\partial \ell\left(x ; \alpha, \lambda_{1}, \lambda_{2}\right)}{\partial \lambda_{1}}=-x_{1 j}+\tau\left(\frac{\lambda_{2}}{\lambda_{1}}\right)^{2}+\alpha \sum_{j=1}^{n_{1}} x_{1 j} t^{*} \\
& +\alpha \tau\left(\frac{\lambda_{2}}{\lambda_{1}}\right)^{2} \sum_{j=1}^{n_{2}} t * *=0 \\
& \frac{\partial \ell\left(x ; \alpha, \lambda_{1}, \lambda_{2}\right)}{\partial \lambda_{2}}=-\left(x_{2 j}-\tau\right)-2 \tau\left(\frac{\lambda_{2}}{\lambda_{1}}\right) \\
& +\alpha \sum_{j=1}^{n_{2}}\left[\left(x_{2 j}-\tau+\tau\left(\frac{\lambda_{2}}{\lambda_{1}}\right)\right)+\alpha \tau\left(\frac{\lambda_{2}}{\lambda_{1}}\right)\right] . t * *=0 . \\
& \text { where } t^{*}=\frac{e^{-\alpha \lambda_{1} x_{1 j}}}{\left(1-e^{-\alpha \lambda_{1} x_{1 j}}\right)} \text { and } t^{* *}=\frac{e^{-\alpha \lambda_{2}\left(x_{2 j}-\tau\left(1-\frac{\lambda_{2}}{\lambda_{1}}\right)\right)}}{\left(1-e^{-\alpha \lambda_{2}\left(x_{2 j}-\tau\left(1-\frac{\lambda_{2}}{\lambda_{1}}\right)\right)}\right)} \text {. }
\end{aligned}
$$

Unfortunately, there is no closed form for $\left(\alpha, \lambda_{1}, \lambda_{2}\right)$ in (3.3), (3.4) and (3.5), so, Newton-Raphson method is used to solve these equations i. It is an iterative method for solving equations $f(\varepsilon)=0$ where $f(\varepsilon)$ is assumed to have a continuous derivative $f^{\prime}(\varepsilon)$. Given a function $f(\varepsilon)$ and its derivative $f^{\prime}(\varepsilon)$, a first guess $\varepsilon_{0}$ is initialed. Then, an approximation of $\varepsilon_{1}$ is $\varepsilon_{0}-\frac{f\left(\varepsilon_{0}\right)}{f^{\prime}\left(\varepsilon_{0}\right)}$ and an approximation of $\varepsilon_{2}$ is $\varepsilon_{1}-\frac{f\left(\varepsilon_{1}\right)}{f^{\prime}\left(\varepsilon_{1}\right)}$ and so on for number of iterations $r$ or if $\left|\varepsilon_{r+1}-\varepsilon_{r}\right| \leq \xi$ where $\varepsilon_{r}$ is the $r^{\text {th }}$ estimate. (see Kotz et al (2003).

Now, to construct the asymptotic Fisher information matrix, the second and mixed derivatives of $\ell\left(x ; \alpha, \lambda_{1}, \lambda_{2}\right)$ with respect to $\alpha, \lambda_{1}$ and $\lambda_{2}$ are taken as follows: 


$$
I=\left[\begin{array}{lll}
\frac{\partial^{2} \ell\left(x ; \alpha, \lambda_{1}, \lambda_{2}\right)}{\partial \alpha^{2}} & \frac{\partial^{2} \ell\left(x ; \alpha, \lambda_{1}, \lambda_{2}\right)}{\partial \alpha \partial \lambda_{1}} & \frac{\partial^{2} \ell\left(x ; \alpha, \lambda_{1}, \lambda_{2}\right)}{\partial \alpha \partial \lambda_{2}} \\
\frac{\partial^{2} \ell\left(x ; \alpha, \lambda_{1}, \lambda_{2}\right)}{\partial \lambda \partial \alpha_{1}} & \frac{\partial^{2} \ell\left(x ; \alpha, \lambda_{1}, \lambda_{2}\right)}{\partial \lambda_{1}^{2}} & \frac{\partial^{2} \ell\left(x ; \alpha, \lambda_{1}, \lambda_{2}\right)}{\partial \lambda_{1} \partial \lambda_{2}} \\
\frac{\partial^{2} \ell\left(x ; \alpha, \lambda_{1}, \lambda_{2}\right)}{\partial \lambda_{2} \partial \alpha} & \frac{\partial^{2} \ell\left(x ; \alpha, \lambda_{1}, \lambda_{2}\right)}{\partial \lambda_{2} \partial \lambda_{1}} & \frac{\partial^{2} \ell\left(x ; \alpha, \lambda_{1}, \lambda_{2}\right)}{\partial \lambda_{2}^{2}}
\end{array}\right] \downarrow\left(\hat{\alpha}, \hat{\lambda}_{1}, \hat{\lambda}_{2}\right)
$$

where,

$$
\begin{aligned}
& \frac{\partial^{2} \ell\left(x ; \alpha, \lambda_{1}, \lambda_{2}\right)}{\partial \alpha^{2}}=\frac{\left(n_{1}+n_{2}\right)(2 \alpha+1)}{\alpha^{2}(\alpha+1)^{2}}-\lambda_{1}^{2} \sum_{j=1}^{n_{1}} x_{1 j}^{2} \cdot t^{*}-\lambda_{1}^{2} \sum_{j=1}^{n_{1}} x_{1 j}^{2} \cdot(t *)^{2} \\
& -\lambda_{2}^{2} \sum_{j=1}^{n_{1}}\left(x_{2 j}-\tau\left(1-\frac{\lambda_{2}}{\lambda_{1}}\right)\right)^{2} \cdot t^{* *} \\
& -\lambda_{2}^{2} \sum_{j=1}^{n_{1}}\left(x_{2 j}-\tau\left(1-\frac{\lambda_{2}}{\lambda_{1}}\right)\right)^{2} \cdot(t * *)^{2} \text {, } \\
& \frac{\partial^{2} \ell\left(x ; \alpha, \lambda_{1}, \lambda_{2}\right)}{\partial \lambda_{1}^{2}}=-\frac{2 \tau}{\lambda_{1}}\left(\frac{\lambda_{2}}{\lambda_{1}}\right)^{2}-\alpha^{2} \sum_{j=1}^{n_{1}} x_{1 j}^{2} t^{*}-\alpha^{2} \sum_{j=1}^{n_{1}} x_{1 j}^{2}(t *)^{2} \\
& -\left[\frac{2 \alpha \tau^{2}}{\lambda_{1}}\left(\frac{\lambda_{2}}{\lambda_{1}}\right)^{2}\right] \sum_{j=1}^{n_{1}} t^{* *}-\left[\alpha^{2} \tau^{2}\left(\frac{\lambda_{2}}{\lambda_{1}}\right)^{4}\right] \sum_{j=1}^{n_{1}} t^{* *} \\
& -\left[\alpha^{2} \tau^{2}\left(\frac{\lambda_{2}}{\lambda_{1}}\right)^{4}\right] \sum_{j=1}^{n_{1}}(t * *)^{2}, \\
& \frac{\partial \ell\left(x ; \alpha, \lambda_{1}, \lambda_{2}\right)}{\partial \lambda_{2}^{2}}=-\frac{2 \tau}{\lambda_{1}}-\left(\frac{2 \alpha \tau}{\lambda_{1}^{2}}\right) \sum_{j=1}^{n_{1}} t * * \\
& -\left(\alpha\left(x_{2 j}-\tau\left(1-\frac{\lambda_{2}}{\lambda_{1}}\right)\right)+\frac{\alpha \tau \lambda_{2}}{\lambda_{1}}\right)^{2} \sum_{j=1}^{n_{1}} t * * \\
& -\left(\alpha\left(x_{2 j}-\tau\left(1-\frac{\lambda_{2}}{\lambda_{1}}\right)\right)+\frac{\alpha \tau \lambda_{2}}{\lambda_{1}}\right)^{2} \sum_{j=1}^{n_{1}}(t * *)^{2},
\end{aligned}
$$




$$
\begin{aligned}
\frac{\partial^{2} \ell\left(x ; \alpha, \lambda_{1}, \lambda_{2}\right)}{\partial \alpha \partial \lambda_{1}} & =\frac{\partial^{2} \ell\left(x ; \alpha, \lambda_{1}, \lambda_{2}\right)}{\partial \lambda_{1} \partial \alpha}=\sum_{j=1}^{n_{1}} x_{1 j} \cdot t^{*}-\alpha \lambda_{1} \sum_{j=1}^{n_{1}} x_{1 j}^{2} \cdot t^{*} \\
& -\alpha \lambda_{1} \sum_{j=1}^{n_{1}} x_{1 j}^{2}(. t *)^{2}-\tau\left(\frac{\lambda_{2}}{\lambda_{1}}\right)^{2} \sum_{j=1}^{n_{1}} t^{* *} \\
& -\alpha \lambda_{2} \tau\left(\frac{\lambda_{2}}{\lambda_{1}}\right)^{2} \sum_{j=1}^{n_{1}}\left(x_{2 j}-\tau\left(1-\frac{\lambda_{2}}{\lambda_{1}}\right)\right) \cdot t^{* *} \\
& -\alpha \lambda_{2} \tau\left(\frac{\lambda_{2}}{\lambda_{1}}\right)^{2} \sum_{j=1}^{n_{1}}\left(x_{2 j}-\tau\left(1-\frac{\lambda_{2}}{\lambda_{1}}\right)\right) .(t * *)^{2},
\end{aligned}
$$

$$
\begin{aligned}
\frac{\partial^{2} \ell\left(x ; \alpha, \lambda_{1}, \lambda_{2}\right)}{\partial \alpha \partial \lambda_{2}} & =\frac{\partial^{2} \ell\left(x ; \alpha, \lambda_{1}, \lambda_{2}\right)}{\partial \lambda_{2} \partial \alpha}=\sum_{j=1}^{n_{1}}\left(x_{2 j}-\tau\left(1-\frac{\lambda_{2}}{\lambda_{1}}\right)\right) \cdot t^{* *} \\
& -\sum_{j=1}^{n_{1}}\left[\alpha\left[\left(x_{2 j}-\tau\left(1-\frac{\lambda_{2}}{\lambda_{1}}\right)\right)+\alpha \tau \frac{\lambda_{2}}{\lambda_{1}}\right]\right. \\
& \left.\times \lambda_{2}\left(x_{2 j}-\tau\left(1-\frac{\lambda_{2}}{\lambda_{1}}\right)\right)\right] . t * * \\
& -\sum_{j=1}^{n_{1}}\left[\alpha\left[\left(x_{2 j}-\tau\left(1-\frac{\lambda_{2}}{\lambda_{1}}\right)\right)+\alpha \tau \frac{\lambda_{2}}{\lambda_{1}}\right]\right. \\
& \left.\times \lambda_{2}\left(x_{2 j}-\tau\left(1-\frac{\lambda_{2}}{\lambda_{1}}\right)\right)\right] .(t * *)^{2}
\end{aligned}
$$

$$
\begin{aligned}
\frac{\partial^{2} \ell\left(x ; \alpha, \lambda_{1}, \lambda_{2}\right)}{\partial \lambda_{1} \partial \lambda_{2}}= & \frac{\partial^{2} \ell\left(x ; \alpha, \lambda_{1}, \lambda_{2}\right)}{\partial \lambda_{2} \partial \lambda_{1}}=-\frac{2 \tau}{\lambda_{1}}\left(\frac{\lambda_{2}}{\lambda_{1}}\right)^{2}+2 \alpha \tau \frac{\lambda_{2}}{\lambda_{1}^{2}} \sum_{j=1}^{n_{1}} t^{* *} \\
& +\alpha \tau\left(\frac{\lambda_{2}}{\lambda_{1}}\right)^{2} \sum_{j=1}^{n_{1}}\left[\alpha\left(x_{2 j}-\tau\left(1-\frac{\lambda_{2}}{\lambda_{1}}\right)\right)+\alpha \tau \frac{\lambda_{2}}{\lambda_{1}}\right] . t * * \\
& +\alpha \tau\left(\frac{\lambda_{2}}{\lambda_{1}}\right)^{2} \sum_{j=1}^{n_{1}}\left[\alpha\left(x_{2 j}-\tau\left(1-\frac{\lambda_{2}}{\lambda_{1}}\right)\right)+\alpha \tau \frac{\lambda_{2}}{\lambda_{1}}\right] . t * *
\end{aligned}
$$

\section{Approximate Confidence Interval}

Let $X_{1}, X_{2}, \cdots, X_{n}$ be two independent random samples drawn from Weighted Exponential distributions as Eq. (1.1) with parameters $\left(\alpha, \lambda_{1}, \lambda_{2}\right)$. They are used at different stress values of $Z_{1}, Z_{2}$. Then the maximum likelihood estimation $\hat{\psi}$ of $\psi$ 
is asymptotically normally distributed with mean $\psi$ and variance $\sigma^{2}(\psi)$ under regularity conditions and large sample. It can be constructed as:

$$
P\left[\hat{\psi}-z_{\gamma / 2} \cdot \sigma(\hat{\psi}) \leq \psi \leq \hat{\psi}+z_{\gamma / 2} \cdot \sigma(\hat{\psi})\right]=1-\gamma .
$$

Now, using following equations and confidence level $1-\gamma=0.95$, the lower and upper confidence intervals can be constructed as:

$$
\begin{aligned}
& \hat{\alpha}-z_{\gamma / 2} \cdot \sigma(\hat{\alpha}) \leq \alpha \leq \hat{\alpha}+z_{\gamma / 2} \cdot \sigma(\hat{\alpha}), \\
& \hat{\theta}_{0}-z_{\gamma / 2} \cdot \sigma\left(\hat{\theta}_{0}\right) \leq \theta_{0} \leq \hat{\theta}_{0}+z_{\gamma / 2} \cdot \sigma\left(\hat{\theta}_{0}\right), \\
& \hat{\theta}_{1}-z_{\gamma / 2} \cdot \sigma\left(\hat{\theta}_{1}\right) \leq \theta_{1} \leq \hat{\theta}_{1}+z_{\gamma / 2} \cdot \sigma\left(\hat{\theta}_{1}\right)
\end{aligned}
$$

\section{Simulation Study}

The computer programs MathCAD (2001) is used to obtain numerical illustration for the last theoretical results. A comparison between the three estimators is performed . 1000 samples generated from Weighted exponential distributions with parameters $\left(\alpha, \lambda_{1}\right)$ and $\left(\alpha, \lambda_{2}\right)$ are used, respectively at different values of $Z_{1}, Z_{2}$ with various size samples $n=n_{2},+n_{2}$ in table 1,2 and 3 .

Relative bias (R Bias) $R E=\left|\frac{\hat{\alpha}-\alpha}{\alpha}\right|$, mean square error (MSE) which is $\operatorname{MSE}(\hat{\alpha})=E\left[(\hat{\alpha}-\alpha)^{2}\right]$, and relative error $(\mathrm{RE}) \operatorname{RE}(\hat{\theta})=\frac{\sqrt{M S E(\hat{\alpha})}}{\alpha}$ are calculated. Also, the lower and upper confidence intervals of the acceleration factor and two shape and scale parameters are obtained. The steps of Simulation are:

1- The random samples are generate from cumulative exposure for Weighted exponential distributions as Eq. (2.2) at various values of $Z_{1}, Z_{2}$ with various size samples $n=n_{2}+n_{2}$. Then, calculate $\log \left(\lambda_{i}\right)=\theta_{0}+\theta_{1} Z_{i} ; i=1,2$.

2- For each sample, the non-linear likelihood system (3.3), (3.4) and (3.5) are solved for $\left(\alpha, \lambda_{1}, \lambda_{2}\right)$ using Newton-Raphson method which was illustrated. 
3- The (R Bias), (MSE) and (RE) of the three parameters $\left(\alpha, \lambda_{1}, \lambda_{2}\right)$ are obtained. Also, for different sample sizes, asymptotic variance and covariance matrix of the estimators are obtained.

4- The lower and upper confidence intervals at significant level $\gamma=0.05$ of the two shape and scale parameters are construct.

Now, from Table (1) and (2), note that the (R Bias), (MSE) and (RE) of the three parameters $\left(\alpha, \lambda_{1}, \lambda_{2}\right)$ are decreasing by increasing the sample size $n$. Also, in table (3), the lower and upper confidence intervals of the acceleration factor and two shape and scale parameters are asymptotic, so the length of interval is decreasing by increasing the sample size.

Table (1) Value of R BIAS, MSE and RE for parameters $\theta_{0}, \theta_{1}, \alpha$ at different stress levels and different sample sizes.

\begin{tabular}{|c|c|c|c|c|c|c|c|}
\hline \multirow{2}{*}{$n_{1}+n_{2}$} & \multirow{2}{*}{$\theta_{0}, \theta_{1}, \alpha$} & \multicolumn{3}{|c|}{$Z_{0}=0.3, Z_{1}=0.7$} & \multicolumn{3}{|c|}{$Z_{0}=0.25, Z_{1}=1$} \\
\hline & & R BIAS & MSE & $\mathrm{RE}$ & R BAIS & MSE & $\mathrm{RE}$ \\
\hline \multirow{3}{*}{50} & $\theta_{0}$ & 0.0708 & 0.1988 & 0.0788 & 0.0478 & 0.1348 & 0.0638 \\
\hline & $\theta_{1}$ & 0.7558 & 0.4278 & 0.6508 & 0.9478 & 0.7018 & 0.8328 \\
\hline & $\alpha$ & 0.2428 & 0.1998 & 0.2438 & 0.2998 & 0.3808 & 0.3008 \\
\hline \multirow{3}{*}{60} & $\theta_{0}$ & 0.0228 & 0.0598 & 0.0408 & 0.0378 & 0.1008 & 0.0538 \\
\hline & $\theta_{1}$ & 0.5918 & 0.3188 & 0.5628 & 0.3418 & 0.6798 & 0.8188 \\
\hline & $\alpha$ & 0.2158 & 0.1698 & 0.2178 & 0.2978 & 0.3798 & 0.3048 \\
\hline \multirow{3}{*}{70} & $\theta_{0}$ & 0.0148 & 0.0308 & 0.0288 & 0.0248 & 0.0428 & 0.0338 \\
\hline & $\theta_{1}$ & 0.5908 & 0.2818 & 0.5298 & 0.2868 & 0.5328 & 0.7268 \\
\hline & $\alpha$ & 0.1998 & 0.1328 & 0.2008 & 0.2898 & 0.3558 & 0.2908 \\
\hline \multirow{3}{*}{80} & $\theta_{0}$ & 0.0088 & 0.0298 & 0.0208 & 0.0118 & 0.0388 & 0.0318 \\
\hline & $\theta_{1}$ & 0.5458 & 0.2428 & 0.4928 & 0.2648 & 0.5018 & 0.7048 \\
\hline & $\alpha$ & 0.1748 & 0.1418 & 0.1778 & 0.2768 & 0.3248 & 0.2778 \\
\hline \multirow{3}{*}{90} & $\theta_{0}$ & 0.0028 & 0.0148 & 0.0198 & 0.0018 & 0.0188 & 0.0228 \\
\hline & $\theta_{1}$ & 0.4188 & 0.2328 & 0.4828 & 0.1558 & 0.4288 & 0.6518 \\
\hline & $\alpha$ & 0.1568 & 0.1128 & 0.1638 & 0.2458 & 0.2498 & 0.2468 \\
\hline \multirow{3}{*}{100} & $\theta_{0}$ & -0.0082 & 0.0148 & 0.0368 & -0.0012 & 0.0128 & 0.0188 \\
\hline & $\theta_{1}$ & 0.3298 & 0.1478 & 0.3878 & 0.1418 & 0.0958 & 0.3178 \\
\hline & $\alpha$ & 0.1488 & 0.0958 & 0.1518 & 0.2428 & 0.2458 & 0.2438 \\
\hline
\end{tabular}


Table (2) Value of R BIAS, MSE and RE for parameters $\theta_{0}, \theta_{1}, \alpha$ at different stress levels and different sample sizes.

\begin{tabular}{|c|c|c|c|c|c|c|c|}
\hline \multirow{2}{*}{$n_{1}+n_{2}$} & \multirow{2}{*}{$\theta_{0}, \theta_{1}, \alpha$} & \multicolumn{3}{|c|}{$Z_{0}=0.4, Z_{1}=1$, } & \multicolumn{3}{|c|}{$Z_{0}=0.8, Z_{1}=1$} \\
\hline & & R BIAS & MSE & RE & R BAIS & MSE & RE \\
\hline \multirow{3}{*}{50} & $\theta_{0}$ & 0.08176 & 0.24276 & 0.08776 & 0.11476 & 0.79476 & 0.16376 \\
\hline & $\theta_{1}$ & 0.48276 & 0.83776 & 0.90876 & 0.64876 & 0.64676 & 0.79876 \\
\hline & $\alpha$ & 0.34376 & 0.49876 & 0.34476 & 0.42476 & 0.75676 & 0.42576 \\
\hline \multirow{3}{*}{60} & $\theta_{0}$ & 0.05176 & 0.12576 & 0.06176 & 0.10476 & 0.66576 & 0.15176 \\
\hline & $\theta_{1}$ & 0.40376 & 0.66976 & 0.81276 & 0.50576 & 0.53276 & 0.72576 \\
\hline & $\bar{\alpha}$ & 0.33476 & 0.47476 & 0.33576 & 0.41276 & 0.71276 & 0.41276 \\
\hline \multirow{3}{*}{70} & $\theta_{0}$ & 0.04176 & 0.09376 & 0.05176 & 0.11676 & 0.45576 & 0.11376 \\
\hline & $\theta_{1}$ & 0.36276 & 0.66476 & 0.80976 & 0.41176 & 0.41476 & 0.64076 \\
\hline & $\alpha$ & 0.32376 & 0.44276 & 0.32476 & 0.40676 & 0.69276 & 0.40676 \\
\hline \multirow{3}{*}{80} & $\theta_{0}$ & 0.02676 & 76 & 0.03276 & 0.06176 & 0.38676 & 0.06976 \\
\hline & $\theta_{1}$ & 0.16576 & 0.42476 & 0.64876 & 0.35576 & 0.28176 & 0.53076 \\
\hline & $\alpha$ & 0.26176 & 0.36676 & 0.29476 & 0.40176 & 0.63676 & 0.38976 \\
\hline \multirow{3}{*}{90} & $\theta_{0}$ & 0.02576 & 0.04076 & 0.02376 & 0.05276 & 0.16576 & 0.06376 \\
\hline & $\theta_{1}$ & 0.16276 & 0.37376 & 0.60876 & 0.23476 & 0.24376 & 0.49376 \\
\hline & $\alpha$ & 0.29276 & 0.36376 & 0.29376 & 0.38976 & 0.63376 & 0.38876 \\
\hline \multirow{3}{*}{100} & $\theta_{0}$ & 0.01276 & 0.02176 & 0.03676 & 0.03376 & 0.13576 & 0.06476 \\
\hline & $\theta_{1}$ & 0.06876 & 0.29976 & 0.54576 & 0.16076 & 0.20476 & 0.20176 \\
\hline & $\alpha$ & 0.28976 & 0.29076 & 0.26276 & 0.38176 & 0.61576 & 0.38376 \\
\hline
\end{tabular}

Table (3) Upper and lower bounds of Confidence intervals at significant level 0.05.

\begin{tabular}{|c|c|c|c|c|c|c|c|}
\hline \multirow{2}{*}{$n_{1}+n_{2}$} & \multirow{2}{*}{$\theta_{0}, \theta_{1}, \alpha$} & \multicolumn{3}{|c|}{$Z_{0}=0.4, Z_{1}=1$} & \multicolumn{3}{|c|}{$Z_{0}=0.8, Z_{1}=1$} \\
\hline & & $\mathrm{LL}$ & UL & Length & $\mathrm{LL}$ & UL & Length \\
\hline \multirow{3}{*}{50} & $\theta_{0}$ & 4.75346 & 5.47046 & 0.50346 & 3.90846 & 5.38446 & 1.26246 \\
\hline & $\theta_{1}$ & -0.00154 & 0.88446 & 0.67246 & -0.10354 & 0.98346 & 0.87346 \\
\hline & $\alpha$ & 1.32146 & 2.02846 & 0.49346 & 0.63246 & 1.83646 & 0.99046 \\
\hline \multirow{3}{*}{60} & $\theta_{0}$ & 4.90946 & 5.61546 & 0.49246 & 5.43546 & 6.43246 & 0.78346 \\
\hline & $\theta_{1}$ & -0.02354 & 0.84346 & 0.65346 & 0.14046 & 0.86346 & 0.50946 \\
\hline & $\alpha$ & 1.19846 & 1.87946 & 0.46746 & 0.75846 & 1.81146 & 0.83946 \\
\hline \multirow{3}{*}{70} & $\theta_{0}$ & 4.71646 & 5.40746 & 0.47746 & 4.93446 & 5.82046 & 0.67246 \\
\hline & $\theta_{1}$ & -0.05654 & 0.79546 & 0.63846 & 0.27446 & 0.95946 & 0.47146 \\
\hline & $\alpha$ & 1.17446 & 1.84546 & 0.45746 & 0.80546 & $\begin{array}{l}1.85646 \\
\end{array}$ & 0.83746 \\
\hline \multirow{3}{*}{80} & $\theta_{0}$ & 4.69046 & 5.27846 & 0.37446 & 5.02846 & 5.84846 & 0.61146 \\
\hline & $\theta_{1}$ & 0.68846 & 0.92646 & 0.02446 & 0.17746 & 0.83046 & 0.43946 \\
\hline & $\alpha$ & 1.19346 & 1.85046 & 0.44346 & 0.92446 & 1.84146 & 0.70346 \\
\hline
\end{tabular}




\begin{tabular}{||c||c||c||c|c||c|c|c||}
\hline \hline \multirow{3}{*}{90} & $\theta_{0}$ & 4.73846 & 5.22646 & 0.27446 & 4.71846 & 5.51246 & 0.53546 \\
\cline { 2 - 7 } & $\theta_{1}$ & 0.69446 & 0.92946 & 0.02146 & 0.24946 & 0.88446 & 0.42146 \\
\cline { 2 - 7 } & $\alpha$ & 1.34546 & 1.87946 & 0.32046 & 1.09646 & 1.79746 & 0.48746 \\
\hline \hline \multirow{3}{*}{100} & $\theta_{0}$ & 4.58646 & 5.16746 & 0.36746 & 4.80746 & 5.51646 & 0.49546 \\
\cline { 2 - 7 } & $\theta_{1}$ & 0.75446 & 0.96646 & 0.00154 & 0.31846 & 0.91046 & 0.37846 \\
\cline { 2 - 7 } & $\alpha$ & 1.36046 & 1.89046 & 0.31646 & 1.16046 & 1.85846 & 0.48446 \\
\hline
\end{tabular}




\section{References}

Abd-Elfattah, A. M. and Al-Harbey, A. H. (2010). Inferences for Burr Parameters Based on Censored Samples in Accelerated Life Tests. JKau: Sci., Vol. 22 No. 2. 149-170.

Al-Ghamdi A. S. \& Hassan, A. S. (2009). Optimum Step Stress Accelerated Life Testing for Lomax Distribution. Journal of Applied Sciences Research. 5(12). 2153-2164.

Azzalini, A. (1985). A class of distributions which include the normal ones. Scandinavian. J. Stat. Vol. 12. 171-178.

Chen, Z., Jie, M. \& Zhou, Y. (2006). Estimating the Mean of Exponential Distribution from Step-Stress Life Test. Data Statistics for Industry and Technology. 307-325.

Dharmadhikari, A. \& Monsur M. (2003). A model for step-stress accelerated life testing. Naval Research Logistics. Vol. (50). 841-868.

Donghoon, H. \& Balakrishnan, N. (2010) Inference for a simple step-stress model with competing risks for failure from the exponential distribution under time constraint. Computational Statistics \& Data Analysis. Vol. 54. 2066-2081

Drop, J. R., Thomas, A., Gordon, E., \& Lee, R. (1996). A Bayes Approach to Step-Stress Accelerated Life Testing. IEEE TRANSACTIONS ON RELIABILITY. VOL. 45. 491-498.

Gupta, R. D. \& Kundu, D. (2009). A new class of weighted exponential distributions. Statistics. Vol. 43(6). 621-634.

Kotz, S., Lumelskii, Y. and Pensky, M. (2003). The Stress-Strength Model and Its Generalizations. New Jersey: World Scientific, Inc.

Lee, H. M., Wu, J. W. \& Lei, C. L. (2013). Assessing the Lifetime Performance Index of Exponential Products With Step-Stress Accelerated Life-Testing Data. IEEE Reliability Society. Vol. (99)

Makhdoom, I. (2012). Estimation of $R=p(Y<X)$ for Weighted Exponential Distribution. J. Applied Sci. Vol. 12 (13). 1384 - 1389. 